\title{
The occurrence of planets and other substellar bodies around white dwarfs using K2
}

\author{
L. van Sluijs, ${ }^{1 \star}$ V. Van Eylen,${ }^{1} \dagger$ \\ ${ }^{1}$ Leiden Observatory, P.O. Box 9513, NL-2300 RA Leiden, The Netherlands
}

Accepted XXX. Received YYY; in original form ZZZ

\begin{abstract}
The majority of stars both host planetary systems and evolve into a white dwarf (WD). To understand their post-main-sequence (PMS) planetary system evolution, we present a search for transiting/eclipsing planets and other Substellar Bodies (SBs) around WDs using a sample of 1148 WDs observed by K2. Using transit injections, we estimate the completeness of our search. We place constraints on the occurrence of planets and substellar bodies around white dwarfs as a function of planet radius and orbital period. For short-period $(P<40$ days $)$ small objects, from asteroid-sized to $1.5 R_{\oplus}$, these are the strongest constraints known to date. We further constrain the occurrence of hot Jupiters $(<1.5 \%)$, habitable zone Earth-sized planets $(<28 \%)$, and disintegrating short-period planets $(\sim 12 \%)$. We blindly recovered all previously known eclipsing objects, providing confidence in our analysis, and make all light curves publicly available.
\end{abstract}

Key words: methods: data analysis - occultations - Planetary Systems - (stars:) white dwarfs - planets and satellites: dynamical evolution and stability

\section{INTRODUCTION}

Most Milky Way stars are orbited by planets (Cassan et al. 2012) and 95\% become a WD (Althaus et al. 2010). An average WD has a radius comparable to the Earth. Therefore, an eclipsing Earth-sized planet around a WD has a $\sim 10^{4}$ times larger transit depth than a solar-type star and would be an excellent candidate to observe faint atmospheric features or the first exomoons (Loeb \& Maoz 2013). Furthermore, WDs host a stable ( $\geq 4$ Gyrs) habitable zone (Agol 2011), have a similar photosynthesis-relevant-wavelength integrated flux and DNA weighted UV dose as the Sun (McCree 1971; Fossati et al. 2014), thus making them excellent candidates to host habitable planets.

Nevertheless, no intact planets around WDs have been found so far (Farihi et al. 2005; Mullally et al. 2006, 2008; Kilic et al. 2009; Drake et al. 2010; Faedi et al. 2011; Fulton et al. 2014; Vanderburg et al. 2015; Xu et al. 2015; Sandhaus et al. 2016). This null detection is in agreement with theoretical simulations of PMS planetary system evolution: the inner planets are destroyed due to tides during the stellar AGBphase (Villaver \& Livio 2009; Kunitomo et al. 2011; Mustill \& Villaver 2012; Villaver et al. 2014). The outer planets planets expand their orbits due to stellar mass loss during

^ E-mail: vansluijs@strw.leidenuniv.nl

$\dagger$ E-mail: vaneylen@strw.leidenuniv.nl the AGB-phase (see. e.g. Veras et al. 2016a,b).

However, several indirect observations of remnant planetary systems exist. About 25-50\% of all WDs have metals in their photosphere (Zuckerman et al. 2003, 2010; Koester et al. 2014). Typical metal sinking time scales are of the order of days to Myrs (Wyatt et al. 2014), much shorter than the typical WD age, therefore these WDs are called metal polluted. Additionally, about $4.6 \%$ of all of the WDs host a debris disk (Wyatt et al. 2014). The amount of accreted material matches asteroid-sized objects (Xu \& Jura 2011; Girven et al. 2012; Farihi et al. 2010) and the compostion matches Solar System meteorites and bulk Earth to zeroth order (Jura \& Young 2014). This suggests metal pollution and debris disks originate from material of remnant planetary systems.

Thus, a missing link must exist between these observations and planetary system evolution theory. The most common explanation is scattering of asteroids by unseen planet towards the WD (Bonsor et al. 2011; Bonsor \& Veras 2015; Debes et al. 2012; Dong et al. 2010; Frewen \& Hansen 2014; Payne et al. 2016). This is supported by simulations showing that AGB-phase stellar mass-loss can cause planets to be perturbed inwards during the WD-phase (Veras \& Gaensicke 2014; Payne et al. 2016). The recent discovery of a disintegrating planet around a WD (Vanderburg et al. 2015) provides additional evidence favoring this scenario.

This discovery and the previous arguments suggest short- 
period SBs around WDs might exist. An opportunity presents itself with the K2 mission (Howell et al. 2014), which observed photometry of a significant number of WDs. Here, we present a search for new transiting/eclipsing SBs around 1148 WDs observed in the first 13 campaigns of $\mathrm{K} 2$. We also determine the recovery rate of transiting objects by injecting artificial transit signals and calculating how many injections were successfully recovered by the transit detection algorithm. Combining the number of transiting/eclipsing SBs and the K2 detection probability allows us to constrain the occurrence of planets and other SBs around these stars. In Section 2, we provide an overview of the K2 WD data set. We explain the adopted methods in Section 3. The results are presented in Section 4 and discussed in Section 5. We draw conclusions in Section 6.

\section{DATA}

The dataset used in this work has been collected by the Kepler satellite during campaigns 1-13 of the K2 mission. During these campaigns, many WDs have been observed for various reasons: to detect transiting $\mathrm{SBs}$, to study eclipsing binary stars, to examine magnetic fields, to study WD pulsations and to search for rotational modulation of WDs. Combined, the initial sample of WDs consists of 1610 targets. An overview of included programs is given in Table 1. Amid these programs a wide diversity of target selection methods exists: some programs only selected spectroscopically confirmed WDs where others used photometric colour and/or reduced proper motion cuts. The latter leads to contamination of the sample by non-WDs with similar photometric and proper motion properties. Secondly, some targets have a main-sequence (MS) companion that contributes a significant fraction of the observed flux. Therefore, the total sample has been cross-correlated with a list of high-probability and confirmed white dwarfs (Hermes et al. $2017)^{1}$. Lastly, there are a dozen duplicate objects within the sample. In total we counted 364 non-confirmed WDs, 90 composite WDs and 39 duplicates within the initial sample of 1610 WDs. Combined, this reduces the total sample to 1148 WDs.

All data files (target pixel files) consist of a time series of images of the target. All initial 1610 targets have been observed with long cadence (30 minutes exposure time). Among these, 374 targets have also been observed with short cadence (1 minute exposure time) of which 309 targets are confirmed, non-composite WDs. Since an Earth-sized planet around an average WD has a transit duration of $\sim 2$ minutes (Agol 2011), long cadence transits are diluted. Therefore, smaller transiting/eclipsing objects can potentially be detected in the short cadence data.

\section{METHODS}

Transiting/eclipsing Stellar and Substellar Bodies (SSBs) cause a periodic drop of the total stellar flux. This research

1 An updated list of K2 confirmed and high-probability candidate WDs can be found at: http://www.k2wd.org (J. J. Hermes 2017, private communication).
Table 1. All included programs constituting the total sample of 1610 targets.

\begin{tabular}{lr}
\hline Campaign & Programs \\
\hline 1 & GO0122, GO0110, GO0071, GO0010, GO0001, \\
2 & GO1071, GO1048, GO1012, GO1007, GO1004 \\
3 & GO02111, GO2087 \\
4 & GO3116, GO3111, GO3087, GO3005 \\
5 & GO4073, GO4043, GO4041, GO4017, GO4003, \\
GO4001 \\
6 & GO6083, GO6063, GO6050, GO6045, GO6003, \\
& GO6001 \\
7 & GO7063, GO7050, GO7045, GO7003, GO7001 \\
8 & GO8048, GO8019, GO818, GO8011, GO8006 \\
9 & \\
10 & GO10006, GO10902, GO10076, GO10048, \\
11 & GO10019, GO10018, GO10011, GO10006 \\
12 & GO11040 \\
13 & GO12901, GO12040, GO12037, GO12027 \\
& GO12007 \\
& GO13040, GO13037, GO13027, GO13007 \\
\hline
\end{tabular}

aims to detects these signals and to evaluate their nature. The initial total sample of 1148 WDs was analysed for this purpose. The amount of SSBs in the data set constrains their occurrence. In Section 3.1, we describe how the pixel target files are converted into light curves. In Section 3.2, we discuss the transit/eclipse detection algorithm, and in Section 3.3, we explain the transit injection procedure, which is necessary to determine the recovery rate of K2 transits. Finally, in Section 3.4 we describe how to combine the transit search with the recovery rate to constrain the transit occurrence.

\subsection{Conversion of raw data into light curves}

To have full control over our analysis and injection pipeline, we start from the raw data, in the form of target pixel files, which can be downloaded from the MAST archive ${ }^{2}$. We then convert the target pixel files into light curves using aperture photometry, and correct for systematic-errors, such as those caused by the pointing jitter in the two-wheel K2 satellite (Howell et al. 2014). Our pipeline to do this is largely based upon the publicly available pipeline ${ }^{3}$ by Van Eylen et al. (2016) and to a lesser extent on other previous research (see e.g. Lund et al. 2015; Vanderburg et al. 2015; SanchisOjeda et al. 2015). We optimized our pipeline for detection of objects around WDs, and describe its main characteristics here $^{4}$ :

(i) Initial flagging: A fraction of the data is flagged using the QUALITY $\operatorname{tag}^{5}$ available for every pixel target file. This describes when special spacecraft events occurred or when

\footnotetext{
2 https://archive.stsci.edu/k2/

3 https://github.com/vincentvaneylen/k2photometry

4 The pipeline is publicly available at https://github.com/ lennartvansluijs/WD-pipeline-K2.

5 More information on the QUALITY tag can be found in the Kepler archive manual http://archive.stsci.edu/kepler/ manuals/archive_manual.pdf
} 
the pipeline flagged a phenomenon. Most of these cannot be triggered by a transit/eclipse signal and are removed. Non-removed tags are: 0 (no event noted), 512 (unused bit by Kepler), 2048 (impulsive outlier before co-trending) and 136426 (local detector electronics parity errors). Trial-anderror found the last tag (2048) to flag in-transit data points and therefore is kept. Tag 136426 mostly affects campaigns 2 and 11 , but for these campaigns $\mathrm{K} 2$ data release notes state the data quality is not adversely affected.

(ii) Aperture photometry: Initially, for the long cadence, irregular apertures are created, starting from the brightest pixel in the concatenated pixel target file and by subsequently adding the brightest neighbouring pixel to the previous aperture, until a maximum of 25 pixels. Using all 25 apertures, the data is reduced in accordance to the description below and the aperture that results in the minimal standard deviation in the flux of the final light curve is chosen as the preferred light curve. For the short cadence observations, the best aperture from the long cadence observations is chosen, because creating 25 apertures for these data is time consuming. For campaigns $>2$ background subtraction is performed already by the $\mathrm{K} 2$ data reduction pipeline. For earlier campaigns, the background flux is estimated for all images in the pixel target file by the median flux of all pixels outside of the best aperture, and this value is subtracted. Finally, we add all flux within the best aperture for the background-removed images to generate the raw light curve. Any 'inf', 'NaN' or 'zero' values are flagged and the light curve is divided by the median.

(iii) Thruster event removal: Every $\sim 6 \mathrm{~h}$, the K2 satellite fires thrusters to maintain its pointing. These events lead to flux outliers and must be removed. To do this, the center-of-flux is calculated for all images in the pixel target file. The differences between subsequent center-of-fluxes are calculated. For the long cadence two data points around differences larger than $3 \sigma$ are removed. For the short cadence, where thruster events affect multiple data points, five data points around differences larger than $3.5 \sigma$ are removed.

(iv) Self-flat-fielding: To separate long-term $\geq 24 \mathrm{~h}$ photometric variations from Kepler's slow drift every $\sim 6.5 \mathrm{~h}$, self-flat-fielding is performed. The data is sliced into parts of $\sim 24 \mathrm{~h}$ and binned. A B-spline is fitted to the binned data, with $3 \sigma$-outliers flagged to ensure a good fit. Division by the best fit decorrelates for the long-term variations.

(v) Drift correction: Firstly, the data set is sliced into 11 parts $(\sim 6.5$ days per part), a timescale for which the satellite's drift has been observed to be quite stable along its roll axis. Secondly, for every slice a principal components analysis is performed to change to a coordinate system where the star moves only along the main direction. A fourth degree polynomial as a function of the moving coordinate is fitted to every slice, with $2 \sigma$-outliers flagged to ensure a good fit. The fluxes are decorrelated against the moving coordinate by division of the best fit. The processes of self-flat-fielding and drift correction are iterated three times to ensure both converted.

All 1148 long cadence and 309 short cadence systematic- error-corrected light curves have been made publicly available $^{6}$.

\subsection{The transit detection algorithm}

After converting the raw data into light curves, transit/eclipse signals must be found. For the long cadence, transit/eclipse dilution causes these signals to become very boxlike. Therefore, an algorithm optimized to search for boxlike signals is used: the Box-Least-Square (BLS) algorithm (Kovács et al. 2002). We used a Python implementation of this algorithm ${ }^{7}$. The BLS algorithm folds a light curve for $n_{P}$ periods within the range $\left(P_{\min }, P_{\max }\right)$, bins the data into $n_{B}$ bins, fits boxes with relative widths within $\left(q_{\min }, q_{\max }\right)$ and calculates the signal-residue $S R(P)$, the BLS-spectrum. Here $n_{P}=10,000$ and $n_{B}=300$ is used, which have sufficient sampling and short computational time. We choose $P_{\text {min }}=1 \mathrm{~h}$, well-below the Roche radius for giants $\sim 5 \mathrm{~h}$ (Fulton et al. 2014). $P_{\max }$ is set to half the total campaign length, i.e. approximately 40 days. Finally, we choose $q_{\min }=10^{-4}$ and $q_{\max }=0.05$, corresponding to a $5 \mathrm{~h}$ Earth $(\sim \operatorname{minimal}$ duration) and a $80 \mathrm{~d}$ Jupiter ( maximal duration) around a WD.

From the BLS-spectrum, three best candidate periods are selected. The first candidate corresponds to the BLS-spectrum maximum. The second and third candidate correspond to the second and third BLS-spectrum maximums, under the extra condition that they are no harmonics of the best candidate period or each other. Harmonics $H$ are defined as periods $P$ for which $\left\{H: \frac{P}{n}, \frac{P}{n-1}, \ldots, P, \ldots,(n-1) P, n P \in\right.$ $(H-H \Delta H, H+H \Delta H)\}$. Here $\Delta H=2 \%$ and $n=11$ are used to ensure candidate periods are not degenerate, but not to exclude other periods.

An overview figure is generated containing the unfolded systematic-error reduced light curve, the BLS-spectrum and three folded systematic-error reduced light curves. All 1148 targets were manually inspected for transit/eclipse signals. Those with large signal-residues for the candidate periods were inspected with extra care, since leftover correlation due to Kepler's pointing jitter can also induce large signalresidues. Furthermore, all targets were inspected for contaminant stars, especially for those where an eclipse/transit signal is detected: these targets were additionally reduced using manually defined apertures encircling only the target star, to ensure the signal does not originate from a nearby field star.

\subsection{Transit injection}

The observed occurrence equals the number of SSBs $n$ over the total sample size $N$. However, this is not the true occurrence: not all SSBs cause an eclipse/transit or are recovered by Kepler. The true occurrence equals $n / N^{\prime}$ where $N^{\prime}$ is the effective sample size defined as

$N^{\prime}\left(P, R_{\mathrm{p}}\right)=p_{\text {tra }}\left(P, R_{\mathrm{p}}\right) \times p_{\text {det }}\left(P, R_{\mathrm{p}}\right) \times N$,

6 The reduced data is publicly available at https://github.com/ lennartvansluijs/WD-pipeline-K2.

7 https://github.com/dfm/python-bls, by Ruth Angus and Dan Foreman-Mackey. 
where $p_{\text {tra }}$ is the transit probability, $p_{\text {det }}$ the detection efficiency (sometimes referred to as coverage or completeness), $P$ the orbital period and $R_{\mathrm{p}}$ the SB radius. The transit probability is fully constrained by the geometry and equals

$p_{\text {tra }}=\frac{R_{\mathrm{p}}+R_{\mathrm{WD}}}{a}$,

where $R_{\mathrm{WD}}$ is the WD radius and $a$ the orbital semi-major axis. $a$ is determined from Kepler's third law assuming a WD mass of $0.6 \mathrm{M}_{\odot}$ and radius $0.012 \mathrm{R}_{\odot}$ (following Fulton et al. 2014).

The detection efficiency depends on both the data quality and the data reduction and is therefore hard to theorize. It can be empirically determined using transit injection. This method injects an artificial transit/eclipse signal into the raw light curve for a given period and SB radius. We use the Mandel and Agol model (Mandel \& Agol 2002) and inject transit signals just before systematic-error-correction (in between step (ii) and (iii) of Section 3.1). If the right period or an harmonic is recovered, one assumes the same would hold for a real transit/eclipse signal.

Firstly, the $\left(P, R_{p}\right)$-plane is divided into 35 tiles by a grid of 5 logarithmically-equally spaced periods between $1 \mathrm{~h}-40 \mathrm{~d}$ and 7 logarithmically-equally spaced radii between 0.125 - $16 R_{\oplus}$. For all injections, a WD of $0.6 \mathrm{M}_{\odot}$ and radius $0.012 \mathrm{R}_{\odot}$ is assumed again. The ellipticity is assumed to be zero, since strong tides are theorized to circularize SB orbits (Nordhaus \& Spiegel 2012; Mustill et al. 2013). The period and planet radius per injection are picked randomly within the tile range. The impact parameter $b$ is chosen uniformly within the transit range $\left(0, b_{\max }\right)$ where

$b_{\max }=\frac{R_{\mathrm{WD}}+R_{p}}{R_{\mathrm{WD}}}$.

For each injection, we pick a random data file from our sample, and we choose a random transit epoch. Many injections per tile must be performed for the detection efficiency to converge to a mean value: 250 injections/square are performed for the long cadence and 110 injections/square for the short cadence, where the computation time is much longer. To ensure the detection efficiency truly represents the K2 detection efficiency for a mixed sample of authentic WDs, injections have been performed only in the sample of 1148 confirmed WDs of which 309 were also observed in short cadence. The detection efficiency equals the recovered injections over the total injections per tile.

\subsection{Constraining the occurrence}

The SSB detection or null-detection combined with the effective sample size constrains the occurrence. A method similar to Faedi et al. (2011) is used here.

Define the fraction of WDs with a SB of radius $R_{\mathrm{p}}$ ), at period $P$ as $f\left(P, R_{\mathrm{p}}\right)$. The probability of finding $n$ SSBs in a sample of $N$ WDs, assuming all SSBs are detectable, is given by a Binominal probability distribution (see e.g. Burgasser et al. 2002; McCarthy \& Zuckerman 2004)

$p(n ; N, f)=\left(\begin{array}{l}N \\ n\end{array}\right) f^{n}(1-f)^{N-n}$.

Not all planets are detectable and $N$ must be replaced by the effective sample size $N^{\prime}$. In the general case of nonzero detections, the occurrence is constrained to the interval $\left(f_{\min }, f_{\max }\right)$ at a confidence level $C$ when satisfying

$\int_{f_{\min }}^{f_{\max }}\left(1+N^{\prime}\right)^{-1} \times p\left(n ; N^{\prime}, f\right)=C$,

where $\left(1+N^{\prime}\right)^{-1}$ is a normalization factor. In the special case of a null detection $n=0$ and $f_{\min }=0$

$\int_{0}^{f_{\max }}\left(1+N^{\prime}\right)^{-1} \times p\left(0 ; N^{\prime}, f\right)=1-\left(1-f_{\max }\right)^{N^{\prime}+1}=C$,

which can be solved for $f_{\max }$

$f_{\max }=1-(1-C)^{\frac{1}{N^{\prime}+1}}$,

calculating the maximum occurrence $f_{\max }$ at a confidence level $C$ for a null detection.

Since the short cadence data has a better detection efficiency than the long cadence, the best effective sample size is the combined long cadence and short cadence effective sample size defined as

$N_{\text {com }}^{\prime}=N_{\mathrm{SC}}^{\prime}+\left(N_{\mathrm{LC}}-N_{\mathrm{SC}}\right) \times p_{\text {tra, } \mathrm{LC}} \times p_{\text {det, } \mathrm{LC}}$.

This can be plugged into Equations 5 or 7 to calculate the occurrence constraints similarly.

\section{RESULTS}

The results are composed of three main components: Firstly, Section 4.1 gives the final list of detected eclipsing/transiting SSBs. Secondly, Section 4.2 discusses the transit injection results. Finally, Section 4.3 presents the occurrence constraints.

\subsection{Detected eclipsing/transiting SSBs}

In total, 10 eclipsing/transiting SSBs have been detected, of which 4 were also observed in short cadence. An overview of the folded light curves is shown in Figure 1. Many of these eclipsing/transiting objects were already known preK2 and selected to be re-observed by K2 to study their properties in more detail. All others were discovered earlier in K2 campaigns. All previously known eclipsing/transiting SSBs detectable in the K2 data sample have been blindly re-obtained, adding confidence to the null detection of new SBs.

The disintegrating planet discovered by Vanderburg et al. (2015), EPIC 201563164, is re-obtained. EPIC 201649211 and 210659779 are both spectroscopically confirmed WDWD binary systems (see e.g. Girven et al. 2012; Silvotti et al. 2012) and EPIC 201371836, 211934173, 246002682 and 251455483 are all spectroscopically confirmed M dwarfWD binary systems (see e.g. Silvestri et al. 2006; Heller et al. 2009; Rebassa-Mansergas et al. 2009). Recently, EPIC 201283111 was tentatively concluded to be the shortestperiod pre-Cataclysmic-Variable star known today (Rappaport et al. 2017; Parsons et al. 2017). Parsons et al. (2017) additionally present EPIC 248368963, likely a WD-brown dwarf binary, but due to dilution of the eclipse there is only slight evidence of an eclipse in the K2 data. Such objects are not recovered by our transit injection algorithm and therefore this object has not been included in our list here. EPIC 

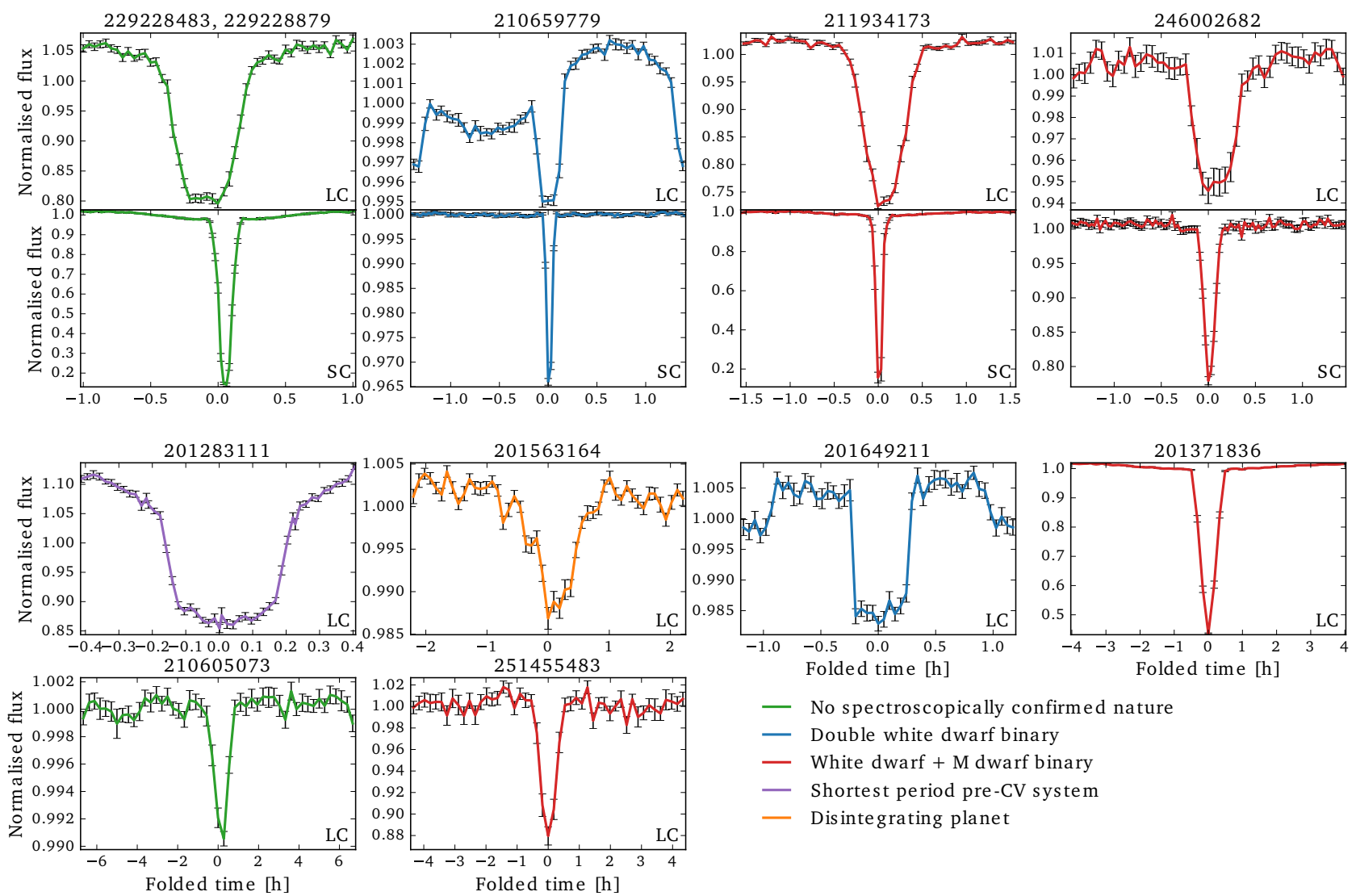

- No spectroscopically confirmed nature

- Double white dwarf binary

- White dwarf + M dwarf binary

— Shortest period pre-CV system

- Disintegrating planet

Figure 1. An overview of the detected eclipsing/transiting SSBs. All light curves are folded for the best period found by the BLSalgorithm. The data is binned after folding using 50 bins for the long cadence ('LC') data and 100 bins for the short cadence ('SC'). The error bars indicate the standard deviation per bin. The colors indicate the natures of the systems.

210605073 was a proposed WD candidate, but a recent photometric analysis suggests the star is (contaminated with) an F0 companion (Adams et al. 2016). The companion may not be a planet: it likely has a significant volatile component, Roche-lobe overflow and/or photo-evaporative mass loss (Adams et al. 2016). The same presumably applies to the previously observed companion around EPIC 229228483 (Kleinman et al. 2004).

\subsection{Transit injection results}

The detection efficiency for the long cadence and short cadence are shown in Figure 2. The error bars indicate the error due to a finite amount of injections and are calculated with bootstrap re-sampling. Bootstrap re-sampling draws $n$ observations with replacement from the original series to create a bootstrap re-sampled series $\left\{P_{i}^{*}\right\}_{j}$ used to determine the re-sampled detection efficiency $p_{\text {det, } \mathrm{j}}^{*}$. This process is iterated $B=10,000$ times and outputs a bootstrap resampled detection efficiency distribution $p_{\text {det, } 1}^{*}, \ldots, p_{\text {det, } \mathrm{B}}^{*}$. The standard deviation $\sigma$ of this distribution measures the error due to a finite amount of injections. The short cadence errors are larger due to less injections.

Large periods have fewer observed transits and smaller SBs have smaller transit depths, causing a lower detection probability. For the long cadence, objects bigger than $1 R_{\oplus}$ for periods $<16 \mathrm{~h}$ are more often recovered than not recovered.
Smaller SBs can be detected in the short cadence data. For the short cadence, objects bigger than $0.5 R_{\oplus}$ for periods $<16 \mathrm{~h}$ are more often recovered than not recovered. Outside these ranges recovery rates are much lower, but still some injections are recovered. This reflects the diversity of the data quality and data reduction in the sample of WDs observed by K2. Overall, Figure 2 shows that K2 is an excellent survey when searching for transiting SSBs around WDs, with the potential of detecting (sub-)Earth-sized planets around WDs.

\subsection{The occurrence constraints}

No non-disintegrating planets and other SBs have been found, therefore Equation 7 is used to constrain their occurrence. Figure 3 presents the occurrence constraints, using the combined long and short cadence effective sample size. The error bars are the propagated transit probability and detection probability errors due to the finite transit injections. Using the basic rule of error propagation $f\left(x_{1}, \ldots, x_{N}\right)=\sqrt{\sum_{i=1}^{N}\left(\partial f / \partial x_{i}\right) \Delta x_{i}}$ the error on the effective sample size equals

$\sigma_{\mathrm{N}^{\prime}}=\sqrt{\left(p_{\mathrm{det}} \times N \times \sigma_{p_{\text {tra }}}\right)^{2}+\left(p_{\text {tra }} \times N \times \sigma_{p_{\text {det }}}\right)^{2}}$.

Combining the long and short cadence (see Equation 9) $\sigma_{N^{\prime}}^{2}=\sigma_{N_{\mathrm{LC}}^{\prime}-N_{\mathrm{SC}}^{\prime}}^{2}+\sigma_{N_{\mathrm{SC}}^{\prime}}^{2}$ and propagating the error to the 

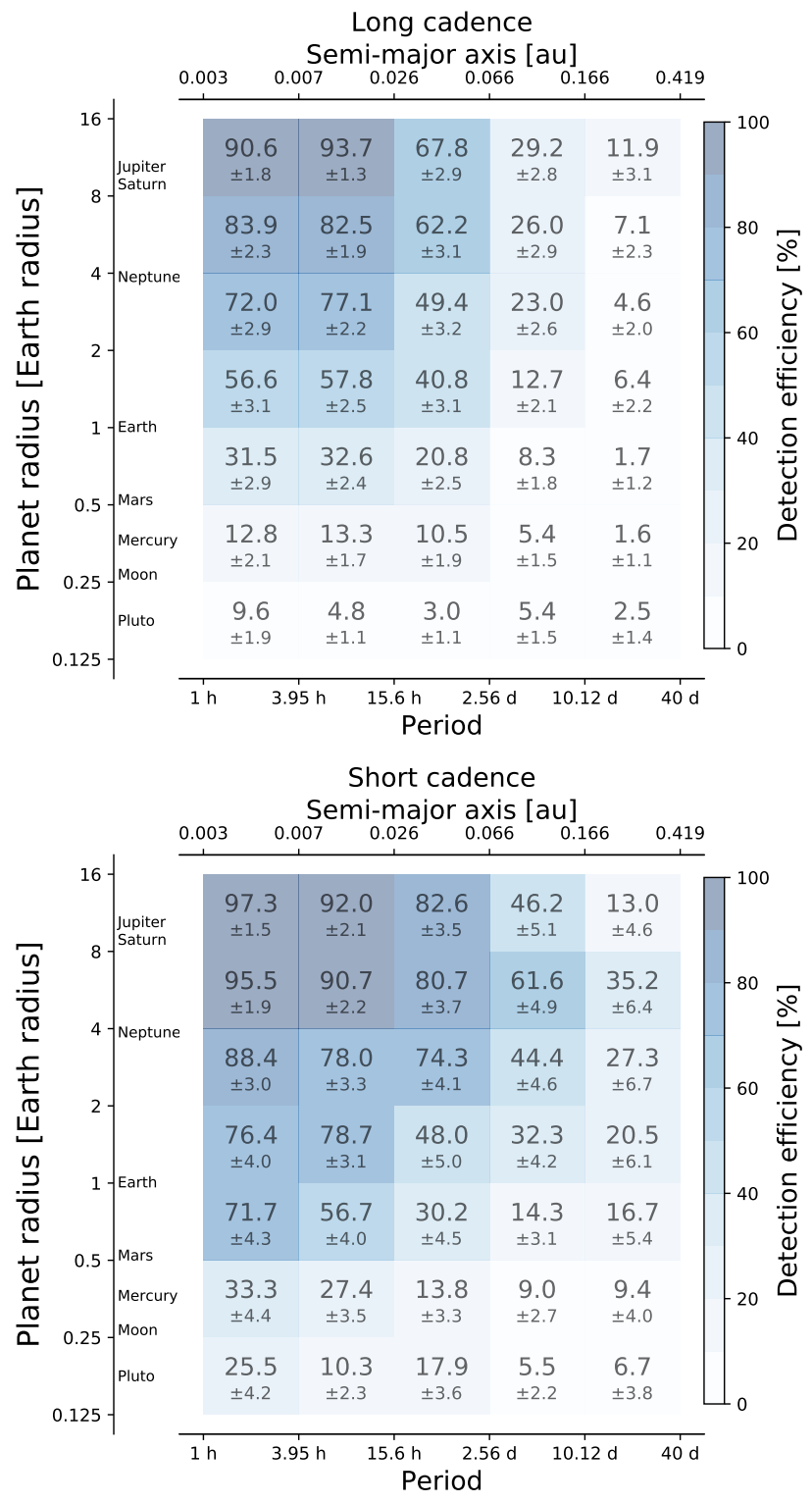

Figure 2. The top panel shows the detection efficiency for the long cadence and the bottom panel for the short cadence. A WD with a mass of $0.6 M_{\odot}$ and a radius of $0.012 R_{\odot}$ is implicitly assumed. The error bars have been calculated by bootstrap re-sampling the injections. Periods and planet radii are logarithmically-equally-spaced. Radii of some solar-system objects are indicated as a comparison.

maximum occurrence equals

$\sigma_{f_{\max }}=\left|\frac{\partial f_{\max }}{\partial N^{\prime}} \sigma_{N^{\prime}}\right|=\left|\frac{\ln (1-C)(1-C)^{1 / N^{\prime}}}{N^{\prime 2}} \sigma_{N^{\prime}}\right|$.

These errors are much smaller than the differences between the two confidence intervals. Equivalently, the finite WD sample size dominantly constrains the maximum occurrence and not the transit injections. Since the transit injection errors are negligible, the errors are dropped from this point. The rest of this work discusses the $95 \%$-confidence interval limits, but the $68 \%$-confidence interval limits are mentioned within brackets.
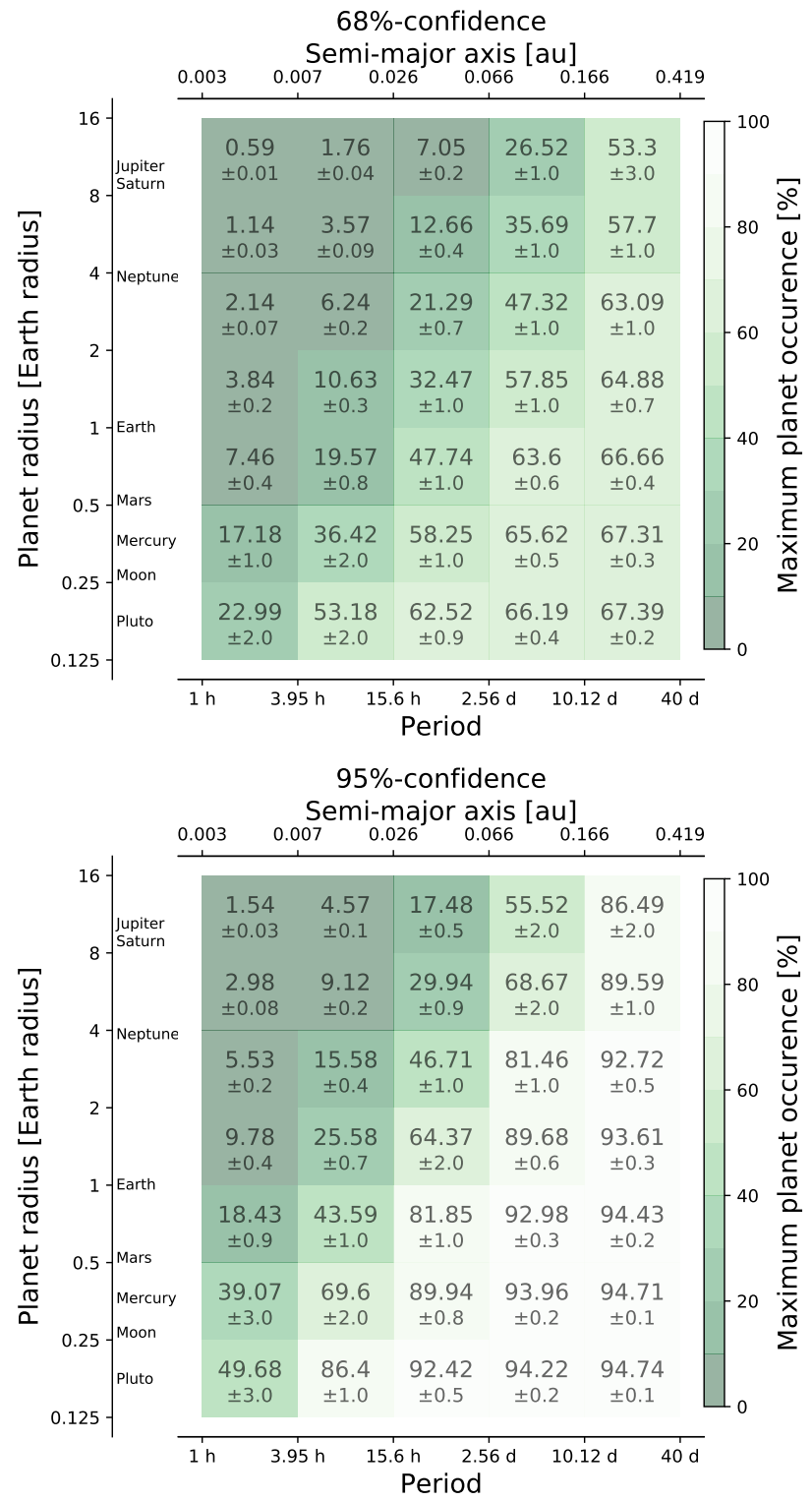

Figure 3. The maximum non-disintegrating SB occurrence around WDs for the combined long and short cadence. The top figure, respectively bottom figure, show the $68 \%$ - interval and a $95 \%$-confidence interval. A WD with a mass of $0.6 M_{\odot}$ and a radius of $0.012 R_{\odot}$ is implicitly assumed. The error bars have been calculated by propagating the finite injection errors of the detection probability and average transit probability. Periods and planet radii are logarithmically-equally-spaced. Radii of some solar-system objects are indicated as a comparison.

We further investigate the occurrence rate of planets in the habitable zone. Agol (2011) defined the Continuous Habitable Zone (CHZ), i.e. the region around an average WD where liquid water is sustainable for $>4$ Gyrs around a WD with mass $0.6 M_{\odot}$ and radius $0.01 R_{\odot}$, close to our radius of $0.012 R_{\odot}$, as having $P=4-32 \mathrm{~d}$, or a semi-major axis $a=0.005-0.02 \mathrm{au}$. We calculate occurrence constraints by selecting only injections within this period range and show the result in Figure 4.

In contrast to the non-disintegrating SB null detection, one 


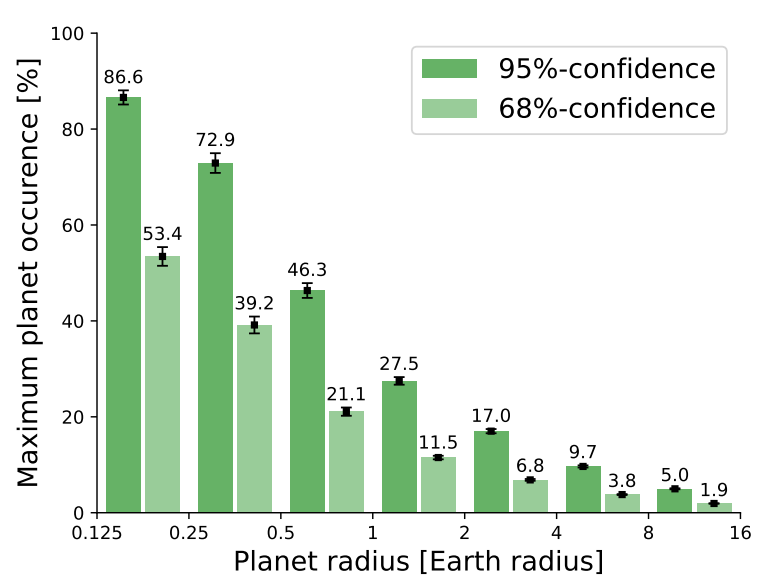

Figure 4. The maximum non-disintegrating SB occurrence around WDs in the CHZ for the combined long and short cadence for a $95 \%$ - and $68 \%$-confidence interval. A WD with a mass of $0.6 M_{\odot}$ and a radius of $0.012 R_{\odot}$ is implicitly assumed. The error bars have been calculated by propagating the finite injection errors of the detection probability and average transit probability. Planet radii are logarithmically-equally-spaced.

detected disintegrating planet exists. Assuming all disintegrating planets have similar transit signals, occurrence constraints can be derived, as done previously by Vanderburg et al. (2015). The transit probability is estimated $p_{\text {tra }} \simeq 0.02$ (Vanderburg et al. 2015). The detection probability is assumed equal to that of a non-disintegrating body transit with a similar transit depth: $p_{\text {det,LC }} \simeq 30 \%$ for the long cadence and $p_{\text {det, SC }} \simeq 57 \%$ for the short cadence. Using these numbers to calculate the effective sample size, a disintegrating planet occurrence of $\sim 12 \%$ is found. To calculate the confidence interval in the general case, Equation 7 is not valid. Instead a Jeffreys interval is used, because its property of being equally-tailed. The obtained $95 \%$ - and respectively $68 \%$-confidence intervals are $\{1 \%, 45 \%\}$ and $\{5 \%, 28 \%\}$.

\section{DISCUSSION}

In Section 5.1, the occurrence constraints are compared to previous research which used surveys other than K2. In Section 5.2, the WD occurrence is compared to the MS occurrence. In Section 5.3, the results are discussed in context with PMS planetary system evolution. Lastly, in Section 5.4 we highlight the caveats and important assumptions of this work.

\subsection{Comparison with previous surveys}

The first attempt to constrain the non-disintegrating SBs occurrence around WDs used data from the Wide Angle Search for Planets (WASP) project (Pollacco et al. 2006) to detect eclipsing SBs (Faedi et al. 2011). They used a smaller WD sample (194) and WASP has a lower sensitivity than K2. Consequently, strong constraints were derived only for short-period gas giants, which occur among $<10 \%$
Table 2. The exoplanetary classification system for WDs used in this work. $\Delta a$ indicates the semi-major axis range, $\Delta P$ the period range and $\Delta R_{p}$ the planet radius range. The ranges are bases on scaled-down versions of the conventional MS classes. An average WD is implicitly assumed.

\begin{tabular}{lccr}
\hline Class & $\begin{array}{c}\Delta a \\
{[\mathrm{au}]}\end{array}$ & $\begin{array}{c}\Delta \boldsymbol{P} \\
{[\mathrm{h}]}\end{array}$ & $\begin{array}{r}\Delta \boldsymbol{R}_{p} \\
{\left[\boldsymbol{R}_{\oplus}\right]}\end{array}$ \\
\hline Hot Jupiters & $<0.005$ & $<4$ & $>10$ \\
Hot (super-)Earths & $<0.005$ & $<4$ & $0.5-2$ \\
Habitable zone (super-)Earths & $0.005-0.02$ & $4-32$ & $0.5-2$ \\
Habitable zone giants & $0.005-0.02$ & $4-32$ & $>2$ \\
Sub-Earth-sized objects & - & - & $<0.5$ \\
\hline
\end{tabular}

of all WDs. The results presented here provide stronger constraints for the full parameter space that was covered.

More recently, the Panoramic Survey Telescope And Rapid Response System (Pan-STARRS) data searched for eclipses around WDs (Fulton et al. 2014). They used a larger WD sample ( 1718 WDs) and Pan-STARSS has equal or better detection probability than $\mathrm{K} 2$ for radii $\geq 2 R_{\oplus}$ for the whole range for which they derived constraints $(0.01-0.04 \mathrm{au})$. Accordingly, they constrained the maximum occurrence of hot Jupiters around WDs $\left(10-20 R_{\oplus}\right.$ in between $0.01-0.04$ au) to be $\leq 0.5 \%$, which is a stronger limit than what is presented here. On the other hand, K2 provides stronger constraints for radii $\leq 2 R_{\oplus}$, particularly for $R \leq 1.5 R_{\oplus}$, which are hardly covered by the ground-based Pan-STARRS survey. K2 most likely has stronger constraints than Pan-STARRS for $>0.04$ au (outside of the range for which Fulton et al. (2014) derived constraints), but the low transit probabilities for WDs make it hard to probe this part of the parameter space even with K2.

\subsection{Comparison with MS planetary systems}

To compare the WD planetary system architecture to that of MS stars the limits in Figure 3 are divided into the more familiar MS classes: hot Jupiters, hot (super-)Earths and habitable-zone (super-)Earths. This is motivated by other studies which showed similarities between MS planetary systems and scaled-down versions of low-mass M-dwarfs planetary systems, the Jupiter-moon system (Muirhead et al. 2012), our solar system and exoplanetary systems around more massive stars (Fulton et al. 2014). Additionally, the classes of habitable-zone giants and sub-Earth-sized objects are added. The inner boundary of the $\mathrm{CHZ}$ is estimated as $0.005 \mathrm{au}(\sim 4 \mathrm{~h})$, 'hot' Jupiters and (super-)Earths are defined to orbit within this boundary. Altogether, the classification adopted in this work is summarized by Table 2 .

Our results constrain the occurrence of hot Jupiters $\leq$ $1.5 \%(0.6 \%)$, which implies hot Jupiters are rare or nonexisting. This is supported by the strong constraints on hot Jupiters by WASP and Pan-STARRS (Faedi et al. 2011; Fulton et al. 2014), and similar or lower than the the occurrence of hot Jupiters around MS stars, which is estimated as $0.3-1.5 \%$ (e.g. Marcy et al. 2005; Gould et al. 2006; Cumming et al. 2008; Howard et al. 2010; Mayor et al. 2011; Wright et al. 2012). Hot (super-)Earths of 1-2 $R_{\oplus}$ occur among $\leq 9.8 \%(3.8 \%)$ of all WDs and for $0.5-1 R_{\oplus}$ among $\leq 18 \%(7.5 \%)$ of all WDs. The occurrence of MS close-in hot 
(super-)Earths is estimated as 23\% (Howard et al. 2010), so that hot (super-)Earths around WDs are likely rarer than around MS stars.

Habitable zone (super-)Earths of 1-2 $R_{\oplus}$ occur among $\leq$ $27.5 \%(11.5 \%)$ of all WDs and for $0.5-1 \quad R_{\oplus}$ among $\leq$ $46.3 \%(21.1 \%)$. Therefore their occurrence is similar or less than their occurrence around MS stars of $\sim 22 \%$ (Petigura et al. 2013). Ice giants $2-4 R_{\oplus}$ in the $\mathrm{HZ}$ are constrained to occur $\leq 17.0 \%(6.8 \%)$ and gas giants are constrained to occur $\leq 5.0 \%(1.9 \%)$.

Small transit probabilities, shorter transit durations and smaller transit depths make non-disintegrating SBs around MS stars $\leq 0.5 R_{\oplus}$ almost undetectable. In contrast to MS stars, K2 can constrain these objects around WDs. In the 'hot' -regime $0.5-1 R_{\oplus}$ occur among $\leq 18.4 \%(7.5 \%)$ of all WDs and for $0.25-0.5 R_{\oplus}$ occur $\leq 39 \%$ (17\%). Even $0.125-0.25 R_{\oplus}$ non-disintegrating SBs can be constrained to occur among $\leq 50 \%(23 \%)$ all WDs.

\subsection{Occurrence constraints in context with PMS planetary system evolution}

The inner planets close to the host star are dragged inwards during the AGB-phase due to tidal interactions and are destroyed (see e.g. Voyatzis et al. 2013). K2 can usefully constrain SBs up to $\sim 0.1$ au. This is well within the region where both gas giants and terrestrial planets are theorized to be destroyed (Mustill \& Villaver 2012). Unless another mechanism exists to migrate outer SBs inwards again, a null detection is thus in good agreement with the theory. Furthermore, the Roche radius for gas giants around WDs is estimated as $L_{\mathrm{R}}=0.01$ au (Fulton et al. 2014). Since $L_{R}>0.005 \mathrm{au}$, the outer boundary for the 'hot' regime, it seems unlikely the analogy of MS hot Jupiters around WDs are sustainable.

The outer planets are less effected by tides and expand their orbits due to the stellar mass loss during the AGB-phase (see e.g. Veras et al. 2016a,b). After the AGB-phase dynamical instabilities can perturb planets (Veras \& Gaensicke 2014) or exomoons (Payne et al. 2016) inwards into the region $\leq 0.1$ au from the WD, the region covered by K2. However, if these SBs remain there just briefly, the probability of detecting one decreases dramatically. Simulation show liberated exomoons orbit within 0.1 au a fraction of $\sim 10^{-3}$ of the full simulation lifetime (Payne et al. 2016, Figure 3). Rigorously assuming all WDs have liberated moons, their occurrence would be $\sim 0.1 \%$, much lower than the limits derived here. The occurrence constraints for larger objects are stronger, but these objects are less likely to orbit close to the WD for a long time (Payne et al. 2016, Figure 3). Nonetheless, these simulations did not take tidal circulization into account (Veras \& Gaensicke 2014; Payne et al. 2016). Tidal circulization around WDs is theorized to occur similarly to MS stars (Mustill et al. 2013). This implies tidal interactions arise from the deformation of the planet rather than the WD. Both Veras \& Gaensicke (2014) and Payne et al. (2016) neglected the interior structure of the planets, for it would complicate their simulations enormously. If tidal circulization successfully stabilizes perturbed planets it could greatly increase the prospects of detecting them.

The estimated disintegrating planet occurrence ( 12\% within $\{1 \%, 45 \%\}$ at $95 \%$-confidence) is most-likely smaller than the fraction of metal polluted WDs of 25-50\% (Cassan et al. 2012). This suggests about half of the metal polluted WDs do not host a disintegrating planet. It remains difficult to interpret these results: the SBs fuelling metal pollution might have been fully disintegrated already, smaller undetectable asteroids may account for the metal pollution or debris disks may fuel the metal pollution instead. Nonetheless, we can conclude disintegrating objects around WDs are most-likely rarer than initially estimated by Vanderburg et al. (2015), due to a larger observed sample with no new such objects detected.

\subsection{Caveats and important assumptions}

The results presented in Figures 2, 3 and 4 are limited by both our data and necessary assumptions. We highlight the caveats and important assumptions of this work:

- The sample size: The size of a WD causes transit probabilities to be much lower for WDs than for MS stars. Therefore, the sample size is the main limiting factor for the occurrence constraints. With only a few more K2 campaigns remaining, the total number of WDs observed by the satellite will not increase dramatically. For the radii larger than $2 R_{\oplus}$ Kepler has a similar detection probability as Pan-STARRS (Fulton et al. 2014). However, since Pan-STARRS has a larger sample size, K2 is outperformed by Pan-STARRS for this part of the parameter space.

- Transit probabilities: the probability for an eclipse rapidly decreases as a function of distance to the star. Therefore, periods $>10 \mathrm{~d}$ are poorly constrained, even though K2 has longer campaign durations of $\sim 80 \mathrm{~d}$.

- K2 data quality and sensitivity: the smallest SBs are poorly constrained due to low detection probability of $\mathrm{K} 2$. Nevertheless, as discussed in Section 5.1, K2 has better detection probability than all currently operating surveys for radii $<1.5 R_{\oplus}$, with some constraints available even for objects as small as $0.125 R_{\oplus}$.

- WD parameters during the transit injection: a WD of mass $0.6 M_{\odot}$ and radius $0.012 R_{\odot}$ was assumed for all transit injections. The vast majority of WDs have parameters close to these, although a small fraction spans a wider range (Tremblay et al. 2016).

- WD contaminant stars: In many cases, WDs are faint stars (for the $\mathrm{K} 2$ sample, the median magnitude is $K_{\mathrm{p}}=$ 18.6), which nearby bright stars may contaminate the light curves and lower the true sensitivity. To mitigate this, WDs with known dM companions were excluded, and pixel files were visually inspected for companion stars.

- Disintegrating planet occurrence constraints: We assumed other disintegrating planets have similar transit signals as EPIC 201563164. However, follow-up observations show the transit depth is highly variable, most-likely due to events of extra dust production by irregular fragmentation of the parent body Gänsicke et al. (2016); Gary et al. (2016). Therefore, we are much more sensitive to disintegrating planets during their active stages compared to their quiescent stages. Additionally, it was assumed the detection efficiency equals that of a non-disintegrating body transit with a similar transit depth. However, their transit signal shapes differ, because of the large dust tail of a disintegrating planet. Therefore, we emphasize taking into account disintegrating planets varying activity levels and proper modelling 
and injection of their light curves and is most-likely necessary to derive more accurate constraints for disintegrating objects around WDs.

\section{CONCLUSIONS}

There is still a lot to learn about the ultimate fate of planetary systems and more observations are favourable. This research analysed 1148 high-probability WDs observed by K2. The WD images were converted into light curves using a new pipeline optimized for WDs observed by K2. A BLS detection algorithm was used to detect transiting/eclipsing objects around the WDs. Ten eclipsing objects were found: the one known disintegrating planet and nine likely stellar objects. From the null detection of new SBs and transit injections, upper limits on the occurrence of planets and SBs were calculated as a function of radius and orbital period. The primary conclusions of this work are:

- At short orbital periods (<40 days), we can constrain the occurrence of small objects around WDs, outperforming previous constraints for asteroid-sized objects up to $1.5 R_{\oplus}$.

- In line with theoretical predictions, hot Jupiters are rare or non-existing and occur among $<1.5 \%$ of all WDs.

- The occurrence of habitable Earth-sized planets (1 $2 R_{\oplus}$ ) around WDs is $<28 \%$, approximately equal or less than their MS occurrence.

- The disintegrating planet occurrence is estimated as $\sim 12 \%$, which is lower than the estimated fraction of metal polluted WDs. However, detailed light curve modelling and taking varying activity levels into account is most-likely necessary to derive more accurate constraints.

- The data reduction and the transit/eclipse detection algorithm used in this work, specifically optimized for WDs observed by K2, successfully retrieved all of the previously known eclipsing objects blindly, adding confidence to the observed null detection of other SBs. The light curves are publicly available.

Further constraining the occurrence of objects orbiting WDs would require larger samples, and any discovery of transiting planets or SBs would be of great interest, because the small size of WDs makes transits ideally suited for e.g. the search for exomoons or atmospheric follow-up studies. More transiting WDs may be detected with the Large Synoptic Survey Telescope (LSST Science Collaboration et al. 2009), TESS (Ricker et al. 2014; Raddi et al. 2017), PLATO (Rauer et al. 2014), NGTS (Wheatley et al. 2013), and Evryscope (Law et al. 2015) .

\section{ACKNOWLEDGEMENTS}

We are grateful to J. J. Hermes for providing his catalogue to crosscheck likely WDs, as well as helpful suggestions that improved this manuscript. We also thank Ignas Snellen for comments on the style and structure of this manuscript.

\section{REFERENCES}

Adams E. R., Jackson B., Endl M., 2016, Astrophysical Journal, 152,47
Agol E., 2011, The Astrophysical Journal, 731, L31

Althaus L. G., Córsico A. H., Isern J., García-Berro E., 2010, The Astronomy and Astrophysics Review, Volume 18, Issue 4, pp.471-566, 18, 471

Bonsor A., Veras D., 2015, Monthly Notices of the Royal Astronomical Society, Volume 454, Issue 1, p.53-63, 454, 53

Bonsor A., Mustill A., Wyatt M., 2011, Monthly Notices of the Royal Astronomical Society, Volume 414, Issue 2, pp. 930939., 414, 930

Burgasser A. J., Kirkpatrick J. D., Reid I. N., Brown M. E., Miskey C. L., Gizis J. E., 2002, The Astrophysical Journal, Volume 586, Issue 1, pp. 512-526., 586, 512

Cassan A., et al., 2012, Nature, Volume 481, Issue 7380, pp. 167169 (2012)., 481, 167

Cumming A., Butler R. P., Marcy G. W., Vogt S. S., Wright J. T., Fischer D. A., 2008, Publications of the Astronomical Society of Pacific, Volume 120, Issue 867, pp. 531 (2008)., 120, 531

Debes J. H., Kilic M., Faedi F., Shkolnik E. L., Lopez-Morales M., Weinberger A. J., Slesnick C., West R. G., 2012, ] 10.1088/0004-637X/754/1/59

Dong R., Wang Y., Lin D. N. C., Liu X. W., 2010, The Astrophysical Journal, Volume 715, Issue 2, pp. 1036-1049 (2010)., 715, 1036

Drake A. J., et al., 2010

Faedi F., West R. G., Burleigh M. R., Goad M. R., Hebb L., 2011, Monthly Notices of the Royal Astronomical Society, 410, 899

Farihi J., Zuckerman B., Becklin E. E., 2005, The Astronomical Journal, 130, 2237

Farihi J., Barstow M. A., Redfield S., Dufour P., Hambly N. C., 2010, Monthly Notices of the Royal Astronomical Society, 404, 2123

Fossati L., et al., 2014, Proceedings of the International Astronomical Union, 10, 325

Frewen S. F. N., Hansen B. M. S., 2014, Monthly Notices of the Royal Astronomical Society, Volume 439, Issue 3, p.2442-2458, 439, 2442

Fulton B. J., et al., 2014, The Astrophysical Journal, Volume 796, Issue 2, article id. 114, 9 pp. (2014)., 796

Gänsicke B. T., et al., 2016, ] 10.3847/2041-8205/818/1/L7

Gary B. L., Rappaport S., Kaye T. G., Alonso R., Hambsch F.-J., 2016, MNRAS, 000, 1

Girven J., Brinkworth C. S., Farihi J., Gänsicke B. T., Hoard D. W., Marsh T. R., Koester D., 2012, The Astrophysical Journal, Volume 749, Issue 2, article id. 154, 11 pp. (2012)., 749

Gould A., et al., 2006, The Astrophysical Journal, Volume 644, Issue 1, pp. L37-L40., 644, L37

Heller R., Homeier D., Dreizler S., Østensen R., 2009, A\&A, 496, 191

Hermes J. J., Gänsicke B. T., Gentile Fusillo N. P., Raddi R., Hollands M. A., Dennihy E., Fuchs J. T., Redfield S., 2017, MNRAS, 468, 1946

Howard A. W., et al., 2010, The Astrophysical Journal, Volume 721, Issue 2, pp. 1467-1481 (2010)., 721, 1467

Howell S. B., et al., 2014, PASP, 126, 398

Jura M., Young E. D., 2014, Annual Review of Earth and Planetary Sciences, 42, 45

Kilic M., Gould A., Koester D., 2009, The Astrophysical Journal, Volume 705, Issue 2, pp. 1219-1225 (2009)., 705, 1219

Kleinman S. J., et al., 2004, The Astrophysical Journal, Volume 607, Issue 1, pp. 426-444., 607, 426

Koester D., Gänsicke B. T., Farihi J., 2014, Astronomy \& Astrophysics, Volume 566, id.A34, 20 pp., 566

Kovács G., Zucker S., Mazeh T., 2002, Astronomy and Astrophysics, v.391, p.369-377 (2002), 391, 369

Kunitomo M., Ikoma M., Sato B., Katsuta Y., Ida S., 2011, The Astrophysical Journal, Volume 737, Issue 2, article id. 66, 8 pp. (2011)., 737 
LSST Science Collaboration et al., 2009, preprint, (arXiv: 0912.0201)

Law N. M., et al., 2015, PASP, 127, 234

Loeb A., Maoz D., 2013, ] 10.1093/mnrasl/slt026

Lund M. N., Handberg R., Davies G. R., Chaplin W. J., Jones C. D., 2015, The Astrophysical Journal, Volume 806, Issue 1, article id. 30, 15 pp. (2015)., 806

Mandel K., Agol E., 2002, The Astrophysical Journal, Volume 580, Issue 2, pp. L171-L175., 580, L171

Marcy G., Butler R. P., Fischer D. A., Vogt S. S., Wright J. T., Tinney C. G., Jones H. R. A., 2005, Progress of Theoretical Physics Supplement, No. 158, pp. 24-42, 158, 24

Mayor M., et al., 2011, eprint arXiv:1109.2497

McCarthy C., Zuckerman B., 2004, The Astronomical Journal, 127,2871

McCree K. J., 1971, Agricultural Meteorology, 9, 191

Muirhead P. S., et al., 2012, The Astrophysical Journal, Volume 747, Issue 2, article id. 144, 16 pp. (2012)., 747

Mullally F., Kilic M., Reach W. T., Kuchner M. J., von Hippel T., Burrows A., Winget D. E., 2006, ] 10.1086/511858

Mullally F., Winget D. E., Degennaro S., Jeffery E., Thompson S. E., Chandler D., Kepler S. O., 2008, Extreme Solar Systems, ASP Conference Series, Vol. 398, proceedings of the conference held 25-29 June, 2007, at Santorini Island, Greece. Edited by D. Fischer, F. A. Rasio, S. E. Thorsett, and A. Wolszczan, p.163, 398, 163

Mustill A. J., Villaver E., 2012, The Astrophysical Journal, Volume 761, Issue 2, article id. 121, 13 pp. (2012)., 761

Mustill A. J., Veras D., Villaver E., 2013, Mon. Not. R. Astron. Soc.XXXX) Printed, 000, 1

Nordhaus J., Spiegel D. S., 2012, Monthly Notices of the Royal Astronomical Society, Volume 432, Issue 1, p.500-505, 432, 500

Parsons S. G., et al., 2017, Monthly Notices of the Royal Astronomical Society, Volume 471, Issue 1, p.976-986, 471, 976

Payne M. J., Veras D., Gaensicke B. T., Holman M. J., 2016, Monthly Notices of the Royal Astronomical Society, Volume 464, Issue 3, p.2557-2564, 464, 2557

Petigura E. A., Howard A. W., Marcy G. W., 2013, Proceedings of the National Academy of Sciences, vol. 110, issue 48, pp. 19273-19278, 110, 19273

Pollacco D. L., et al., 2006, The Publications of the Astronomical Society of the Pacific, Volume 118, Issue 848, pp. 1407-1418., 118,1407

Raddi R., et al., 2017, preprint, (arXiv:1708.09394)

Rappaport S., et al., 2017, MNRAS, 000, 1

Rauer H., et al., 2014, Experimental Astronomy,

Rebassa-Mansergas A., Gaensicke B. T., Schreiber M. R., Koester D., Rodriguez-Gil P., 2009, Monthly Notices of the Royal Astronomical Society, Volume 402, Issue 1, pp. 620-640., 402, 620

Ricker G. R., et al., 2014, in Society of Photo-Optical Instrumentation Engineers (SPIE) Conference Series. p. 20 (arXiv: 1406.0151), doi:10.1117/12.2063489

Sanchis-Ojeda R., et al., 2015, The Astrophysical Journal, 812, 112

Sandhaus P. H., Debes J. H., Ely J., Hines D. C., Bourque M., 2016, ] 10.3847/0004-637X/823/1/49

Silvestri N. M., et al., 2006, The Astronomical Journal, 131, 1674

Silvotti R., et al., 2012, Monthly Notices of the Royal Astronomical Society, 424, 1752

Tremblay P.-E., Cummings J., Kalirai J. S., Gänsicke B. T., Gentile-Fusillo N., Raddi R., 2016, MNRAS, 461, 2100

Van Eylen V., et al., 2016, ApJ, 820, 56

Vanderburg A., et al., 2015, Nature, 526, 546

Veras D., Gaensicke B. T., 2014, Monthly Notices of the Royal Astronomical Society, Volume 447, Issue 2, p.1049-1058, 447, 1049
Veras D., Mustill A. J., Gänsicke B. T., Redfield S., Georgakarakos N., Bowler A. B., Lloyd M. J. S., 2016a, Mon. Not. R. Astron. Soc, 000, 1

Veras D., Mustill A. J., Gänsicke B. T., Redfield S., Georgakarakos N., Bowler A. B., Lloyd M. J. S., 2016b, Mon. Not. R. Astron. Soc, 000, 1

Villaver E., Livio M., 2009, The Astrophysical Journal Letters, Volume 705, Issue 1, pp. L81-L85 (2009)., 705, L81

Villaver E., Livio M., Mustill A. J., Siess L., 2014, The Astrophysical Journal, Volume 794, Issue 1, article id. 3, 15 pp. (2014)., 794

Voyatzis G., Hadjidemetriou J. D., Veras D., Varvoglis H., 2013, Monthly Notices of the Royal Astronomical Society, 430, 3383

Wheatley P. J., et al., 2013, in European Physical Journal Web of Conferences. p. 13002 (arXiv:1302.6592), doi:10.1051/epjconf/20134713002

Wright J. T., Marcy G. W., Howard A. W., Johnson J. A., Morton T. D., Fischer D. A., 2012, The Astrophysical Journal, 753

Wyatt M. C., Farihi J., Pringle J. E., Bonsor A., 2014, ] $10.1093 /$ mnras/stu183

Xu S., Jura M., 2011, The Astrophysical Journal, Volume 745, Issue 1, article id. 88, 14 pp. (2012)., 745

Xu S., Ertel S., Wahhaj Z., Milli J., Scicluna P., Bertrang G. H. M., 2015, Astronomy \& Astrophysics, Volume 579, id.L8, 5 pp., 579

Zuckerman B., Koester D., Reid I. N., Hunsch M., 2003, The Astrophysical Journal, 596, 477

Zuckerman B., Melis C., Klein B., Koester D., Jura M., 2010, The Astrophysical Journal, Volume 722, Issue 1, pp. 725-736 (2010)., 722, 725

This paper has been typeset from a $\mathrm{T}_{\mathrm{E}} \mathrm{X} / \mathrm{LAT}_{\mathrm{E}} \mathrm{X}$ file prepared by the author. 